\title{
NEW UNDERGROUND NEUTRINO OBSERVATORY - GENIUS - IN THE NEW MILLENIUM : FOR SOLAR NEUTRINOS, DARK MATTER AND DOUBLE BETA DECAY
}

\author{
H.V. KLAPDOR-KLEINGROTHAUS \\ Max-Planck-Institut für Kernphysik, \\ P.O. Box 1039 80, D-69029 Heidelberg, Germany \\ Spokesman of HEIDELBERG-MOSCOW and GENIUS Collaborations \\ E-mail:klapdor@gustav.mpi-hd.mpg, \\ Home-page: http://mpi-hd.mpg.de.non_acc
}

\begin{abstract}
Double beta decay is indispensable to solve the question of the neutrino mass matrix together with $\nu$ oscillation experiments. The most sensitive experiment since eight years - the HEIDELBERG-MOSCOW experiment in Gran-Sasso - already now, with the experimental limit of $\left\langle m_{\nu}\right\rangle<0.26 \mathrm{eV}$ excludes degenerate $\nu$ mass scenarios allowing neutrinos as hot dark matter in the universe for the small angle MSW solution of the solar neutrino problem. It probes cosmological models including hot dark matter already now on the level of future satellite experiments MAP and PLANCK. It further probes many topics of beyond Standard Model physics at the $\mathrm{TeV}$ scale. Future experiments should give access to the multi-TeV range and complement on many ways the search for new physics at future colliders like LHC and NLC. For neutrino physics GENIUS will allow to test almost all neutrino mass scenarios allowed by the present neutrino oscillation experiments. At the same time GENIUS will cover a wide range of the parameter space of predictions of SUSY for neutralinos as cold dark matter. Further it has the potential to be a real-time detector for low-energy ( $p p$ and ${ }^{7} \mathrm{Be}$ ) solar neutrinos. A GENIUS Test Facility has just been funded and will come into operation by end of 2001 .
\end{abstract}

\section{Introduction}

Underground physics can complement in many ways the search for New Physics at future colliders such as LHC and NLC and can serve as important bridge between the physics that will be gleaned from future high energy accelerators on the one and and satellite experiments such as MAP and PLANCK on the other $112822,20.1729 .28$.

The first indication for beyond Standard Model (SM) physics indeed has come from underground experiments (neutrino oscillations from $\mathrm{Su}-$ perkamiokande), and this type of physics will play an even large role in the future.

Concerning neutrino physics, without double beta decay there will be no solution of the nature of the neutrino (Dirac or Majorana particle) and of the structure of the neutrino mass matrix. Only investigation of $\nu$ oscillations and double beta decay together can lead to an absolute mass scale1.2. $3.4,23$.

KK-LowNu00: submitted to World Scientific on December 17, 2018 
Concerning solar neutrino physics, present information on possible $\nu$ oscillations relies on $0.2 \%$ of the solar neutrino flux. The total $p p$ neutrino flux has not been measured and also no real-time information is available for the latter. Concerning the search for cold dark matter, direct detection of the latter by underground detectors remains indispensable.

The GENIUS project proposed in 1997-178328,27 as the first third generation $\beta \beta$ detector, could attack all of these problems with an unprecedented sensitivity. The main goals of GENIUS are dark matter search and double beta decay. In this paper we shall concentrate on the neutrino physics with emphasis on solar neutrinos, and on some dark matter aspects. The GENIUS project at this conference is for the first time presented in its capacity of a solar neutrino detector to the community of solar neutrino experts. GENIUS will allow real time detection of low-energy solar neutrinos with a threshold of $19 \mathrm{keV}$. For the further potential of GENIUS for other beyond SM physics, such as SUSY, compositeness, leptoquarks violation of Lorentz invariance and equivalence principle, etc we refer to $29.28,3.20,19$.

\section{GENIUS and Low-Energy Solar Neutrinos}

To solve the solar neutrino problem, a measurement of the solar low-energy spectrum in real time is required. The reasons are: The prediction of the pp neutrino flux is almost solar-model independent and strongly constrained

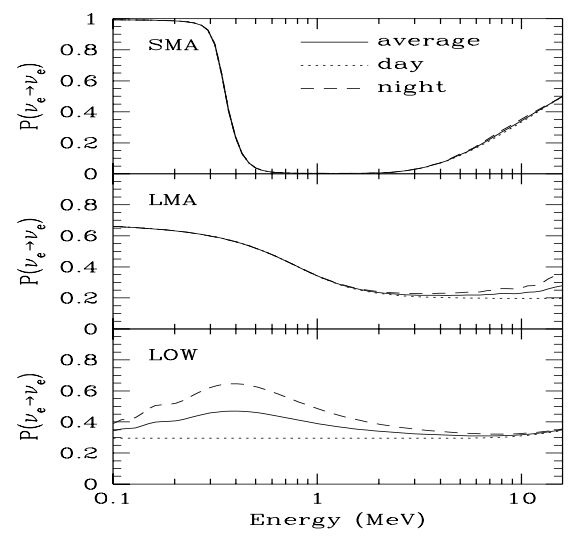

Figure 1. Survival prebabilities for an electron neutrino created in the Sun for the three MSW solutions (from 38). SMA, LMA, LOW stand for the small mixing angle, the large mixing angle and the low $\Delta m^{2}$ MSW solutions. 


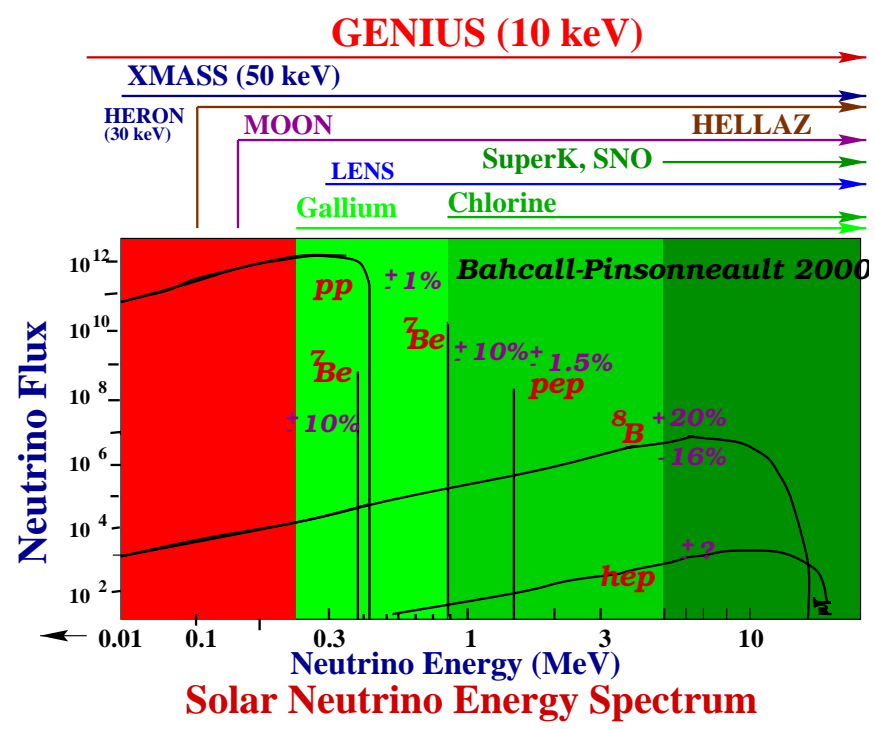

Figure 2. The sensitivity (thresholds) of different running and projected solar neutrino detectors (see 35 and HEIDELBERG NON-ACCELERATOR PARTICLE PHYSICS GROUP home-page: http://www.mpi-hd.mpg.de/non_acc/).

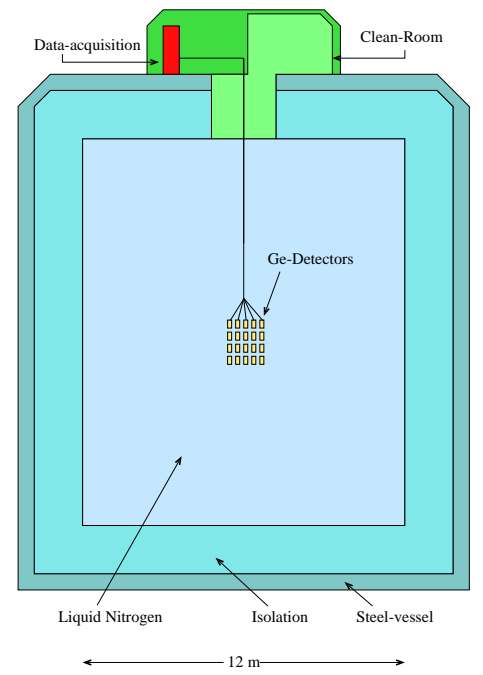

Figure 3. Schematic view of the GENIUS project. An array of $100 \mathrm{~kg}$ of natural HPGe detectors for the WIMP dark matter search (first step) or between 0.1 and 10 tons of enriched ${ }^{76} \mathrm{Ge}$ for the double beta decay search (final setup) is hanging on a support structure in the middle of the tank immersed in liquid nitrogen. The size of the nitrogen shield would be 12 meters in diameter at least. On top of the tank a special low-level clean room and the room for the electronics and data acquisition will be placed. 
by the solar luminosity and helioseismological measurements. The spectral distortion by oscillations allows to differentiate between different oscillation solutions. It is obvious from (Fig. 1) 38 that a threshold below $300 \mathrm{keV}$ is preferable, i.e. lower than achieved by GALLEX and SAGE and also lower than those of some future experiments.

GALLEX and SAGE measure $p p+{ }^{7} \mathrm{Be}+{ }^{8} \mathrm{~B}$ neutrinos $(60+30+10 \%)$ down to $0.24 \mathrm{MeV}$, the Chlorine experiment measured ${ }^{7} \mathrm{Be}+{ }^{8} \mathrm{~B}$ neutrinos $\left(80 \%{ }^{8} \mathrm{~B}\right)$ above $E_{\nu}=0.817 \mathrm{MeV}$, all without spectral, time and direction information. No experiment has separately measured the $p p$ and ${ }^{7}$ Be neutrinos and no experiment has measured the full $p p \nu$ flux. BOREXINO plans to measure ${ }^{7} \mathrm{Be}$ neutrinos in real time, the access to $p p$ neutrinos being limited by ${ }^{14} \mathrm{C}$ contamination (the usual problem of organic scintillators). GENIUS which has been proposed for solar $\nu$ detection in 1999t, could be the first detector measuring the full $p p$ (and ${ }^{7} \mathrm{Be}$ ) neutrino flux in real time (Fig. 2).

The main idea gf GENUS, originally proposed for double beta and dark

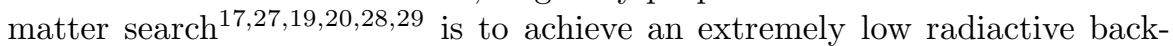
ground (factor of $>1000$ smaller than in the HEIDELBERG-MOSCOW expriment) by using 'naked' detectors in liquid nitrogen (Fig. 3).

While for double beta decay search a detector mass of $0.1-10$ tons of enriched ${ }^{76} \mathrm{Ge}$ is foreseen, and for cold dark matter search $100 \mathrm{~kg}$ of natural Ge detectors are sufficient, GENIUS as a solar neutrino detector would contain 1-10 tons of enriched ${ }^{70} \mathrm{Ge}$ or ${ }^{73} \mathrm{Ge}$.

That Ge detectors in liquid nitrogen operate excellently, has been demonstrated in the Heidelberg low-level laboratory 5 - 9 and the overall feasibility of the project has been shown in 18 .

The potential of GENIUS to measure the spectrum of low-energy solar neutrinos in real time has been studied by 18 . The detection reaction is elastic neutrino-electon scattering $\nu+e^{-} \longrightarrow \nu+e^{-}$. In $\nu$-e scattering experiments relatively higher statistics is achieved then in absolution experiments (see Table 3), but no conicidence information is available as in some of the absorption experiments.

The maximum electron recoil energy is $261 \mathrm{keV}$ for the pp neutrinos and $665 \mathrm{keV}$ for the ${ }^{7} \mathrm{Be}$ neutrino 36 . The recoil electrons can be detected through their ionization in a HP Ge detector with an energy resolution of $0.3 \%$. GENIUS can measure only (like BOREXINO, and others) but with much better energy resolution) the energy distribution of the recoiling electrons, and not directly determine the energy of the incoming neutrinos. The dominant part of the signal in GENIUS is produced by $p p$ neutrinos $(66 \%)$ and ${ }^{7} B e$ neutrinos (33\%). The detection rates for the $p p$ and ${ }^{7} B e$ fluxes are according to the Standard Solar Model39 $R_{p p}=35 \mathrm{SNU}=1.8$ events/day ton $\quad(18$ 


\begin{tabular}{|c|c|c|}
\hline & $\begin{array}{c}\text { Events/day } \\
11-665 \mathrm{keV} \\
(1 \mathrm{ton})\end{array}$ & $\begin{array}{c}\text { Events/day } \\
11-665 \mathrm{keV} \\
(10 \mathrm{tons})\end{array}$ \\
\hline \hline SSM: & 2.4 & 24. \\
$p p:$ & $1.8(35 \mathrm{SNU})$ & 18. \\
${ }^{7} \mathrm{Be}:$ & $0.6(13 \mathrm{SNU})$ & 6. \\
\hline Full $\nu_{e} \rightarrow \nu_{\mu}$ conversion & 0.62 & 6.2 \\
\hline Background & 0.6 & 6. \\
\hline \hline
\end{tabular}

Table 1. Neutrino signal rates in GENIUS for 1 ton (10 tons) of Germanium.

events/day 10 tons) and $R_{7} B e=13 \mathrm{SNU}=0.6$ events/day ton $(6$ events/day 10 tons) ( $1 \mathrm{SNU}=10^{-36} / \mathrm{s}$ target atom).

The expected total number of events, according to the SSM, and for full $\nu_{e} \longrightarrow \nu_{\mu}$ conversion, are given in Table 1 .

The expected spectrum of the low-energy signal in the SSM is shown in Fig. A, together with the total expected background (which will be discussed below).

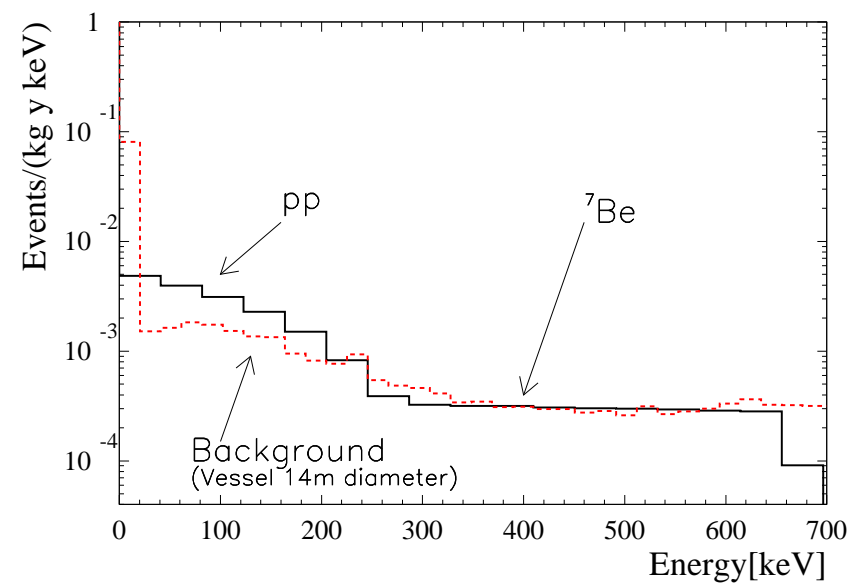

Figure 4. Simulated spectrum of low-energy solar neutring (according to SSM) for the GENIUS detector (1 tonne of natural or enriched Ge) (froml).

Due to the excellent energy resolution of the detectors and the difference in the cross section of electron and muon neutrinos, a comparison of the energy spectrum of recoiling electrons with the theoretical prediction for various $\nu$ 
oscillation scenarios can be made to the following extent. LMA and SMA MSW solutions can be differentiated only to a limited degree. After the unfavouring of the SMA solution by Superkamiokande, it may, however, now be more important to differentiate between the LMA and the LOW solution. Here due to its relatively high counting rate, GENIUS will be able to test in particular the LOW solution of the solar $\nu$ problem by the expected day/night variation of the flux (see Fig. 1).

If the signal to background ratio $\mathrm{S} / \mathrm{B}$ will be greater than 1 , then the $p p$ and ${ }^{7} \mathrm{Be}$ fluxes can be measured by spectroscopic techniques alone. If $\mathrm{S} / \mathrm{B}<1$, one can make use of the seasonal variation of the solar flux ( $7 \%$ from maximum to minimum) related with the eccentricity of the Earth's orbit.

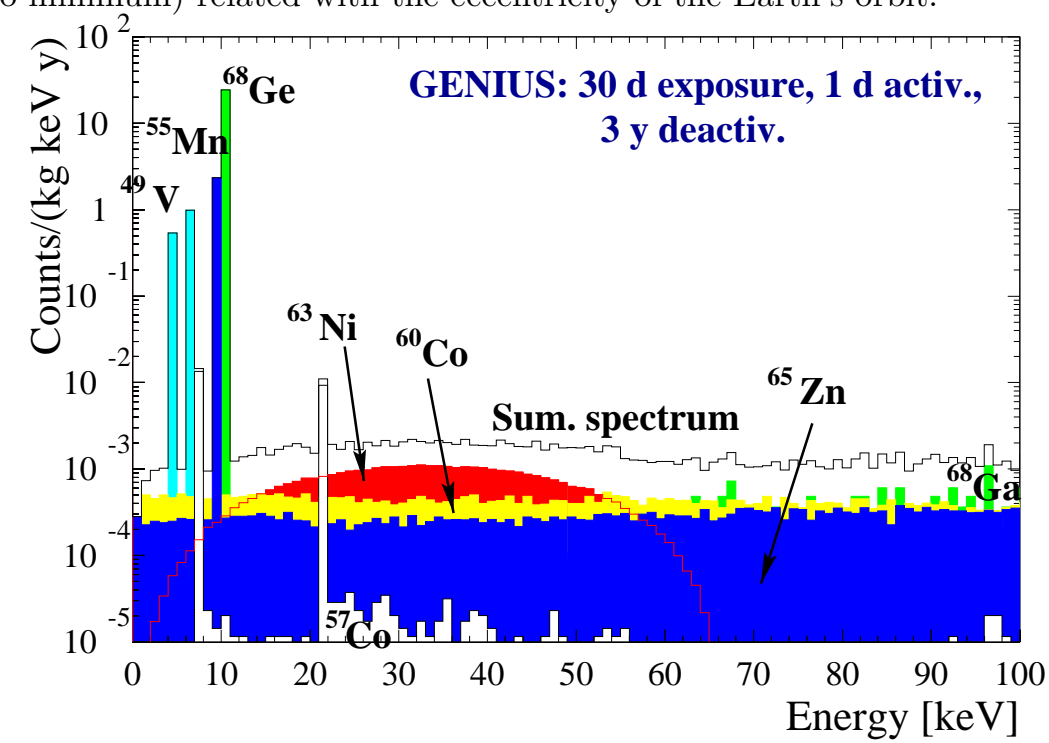

Figure 5. Simulated cosmogenic background during detector production. Assumptions: 30 days exposure of material before processing, $1 \mathrm{~d}$ activatipmafter zone refining, $3 \mathrm{y}$ deactivation underground (neglecting tritium production) (seel, 15 ).

\section{Background Requirements}

The average neutrino-induced signal is $3 \times 10^{-3}$ events $/ \mathrm{kg}$ y keV in the energy region from 0 to $260 \mathrm{keV}$. Thus, to measure the low-energy solar $\nu$ flux with a signal to background ratio of $3: 1$, the required background rate is about $1 \times 10^{-3}$ events $/ \mathrm{kg} \mathrm{y} \mathrm{keV}$ in this energy range. This is about a factor of 10 smaller than what is required for the application of GENIUS for cold dark matter search. This can be achieved if the liquid nitrogen shielding is 


\begin{tabular}{|l|c|c|c|}
\hline Source & Component & Assumption & $\begin{array}{c}\text { events/(kg y keV)] } \\
11-260 \mathrm{keV}\end{array}$ \\
\hline $\begin{array}{l}\text { LNitrogen } \\
\text { contamination }\end{array}$ & ${ }^{238} \mathrm{U},{ }^{232} \mathrm{Th},{ }^{40} \mathrm{~K}$ & $3.5,4.4,10 \times 10^{-16} \mathrm{~g} / \mathrm{g}$ & $3.6 \times 10^{-4}$ \\
${ }^{222} \mathrm{Rn}$ & $0.5 \mu \mathrm{Bq} / \mathrm{m}^{3}$ & $2.5 \times 10^{-5}$ \\
\hline Steel vessel & $\mathrm{U} / \mathrm{Th}$ & $10^{-8} \mathrm{~g} / \mathrm{g}$ & $4.5 \times 10^{-5}$ \\
\hline Holder system & $\mathrm{U} / \mathrm{Th}$ & $10^{-13} \mathrm{~g} / \mathrm{g} ; 13 \mathrm{~g} /$ det. & $8 \times 10^{-5}$ \\
\hline Surrounding & Gammas & GS flux; tank: $13 \times 13 \mathrm{~m}$ & $9 \times 10^{-4}$ \\
& Neutrons & GS flux & $3 \times 10^{-4}$ \\
& Muon shower & GS flux; muon veto $96 \%$ & $7.2 \times 10^{-6}$ \\
& $\mu \rightarrow \mathrm{n}\left({ }^{71} \mathrm{Ge}\right)$ & 230 capt. in nat. Ge/y & $5 \times 10^{-4}$ \\
\hline Cosmogenic & $54 \mathrm{Mn},{ }^{57} \mathrm{Co},{ }^{60} \mathrm{Co}$, & $1 \mathrm{~d}$ activ., $5 \mathrm{y}$ deactiv. & $38 \times 10^{-4}$ \\
& ${ }^{63} \mathrm{Ni},{ }^{65} \mathrm{Zn},{ }^{68} \mathrm{Ge}$ & & $\mathbf{3} \times \mathbf{1 0}^{-3}$ \\
\hline Total & & & \\
\hline
\end{tabular}

Table 2. Simulated background sources together with the made assumptions mnd the resulting event rates in GENIUS for the low-energy region of the spectrum (from

increased to at least $13 \mathrm{~m}$ in diameter and production of the Ge detectors is performed underground. Table 2 shows the result of corresponding Monte Carlo simulations (sed). Regarding the radiactivity of liquid nitrogen, the values reached at present by BOREXINO for their liquid scintillator would be sufficient. The external background from gamma rays from the Gran Sasso laboratory could be further reduced by increasing the tank beyond $13 \mathrm{~m}$, or by further outside shielding.

Most attention has to be given to the cosmogenic activation of the Ge crystals at the Earth surface. In Table 2 an overground activation of only 1 day, and 5 years of deactivation have been assumed. Figure a shows the simulated cosmogenic background during detector production, assuming 30 days of exposure of the material to cosmic rays before detector production, one day of activation after zone refining, and three years of deactivation underground (neglecting tririum production, which would set a lower limit (threshold) of the detector at $\sim 19 \mathrm{keV}$ seet 6 ).

Fig. 6 shows the total background in a $13 \mathrm{~m}$ liquid nitrogen tank for detectors produced as described above, but with a deactivation time of 5 years. Obviously the optimum solution would be to produce the detectors in an underground facility.

Another source of background not included in the above discussion is $2 \nu \beta \beta$ decay of ${ }^{76} \mathrm{Ge}$, which is contained in natural Ge with $7.8 \%$. The expected rate 


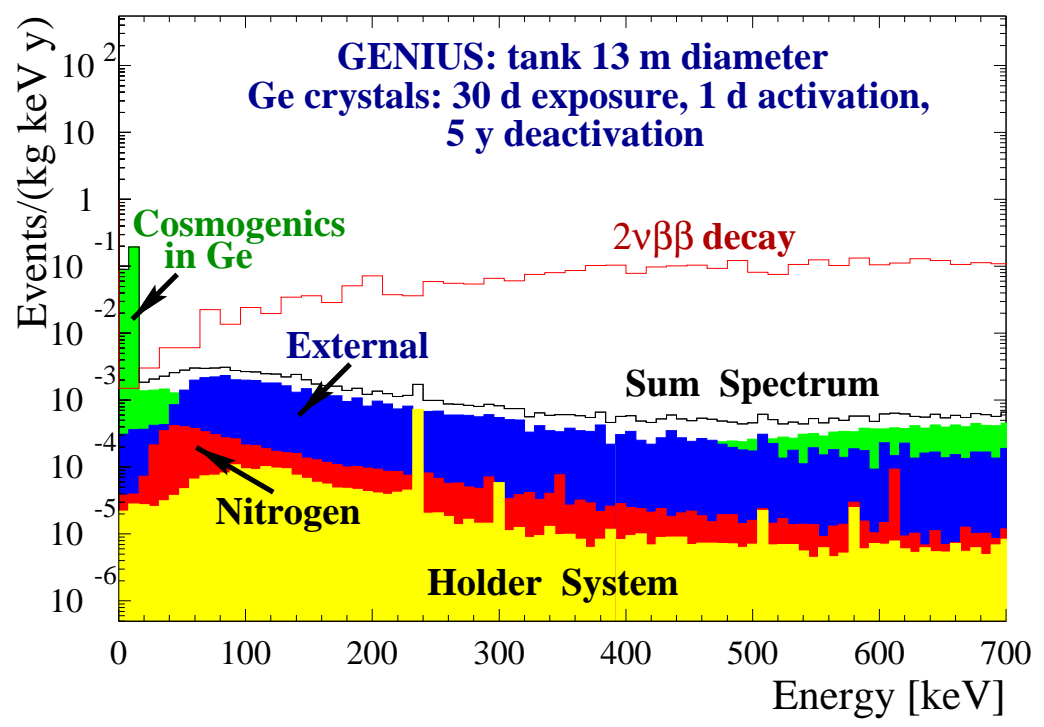

Figure 6. Totalbackground in a $13 \mathrm{~m}$ liqqi nitrogen tank for detectors produced as described in Fig. 5 (tritium neglected) (see 15 ).

for a detector of natural Ge is shown in Fig. 6. The signal to $2 \nu \beta \beta$ background ration for the $p p$ neutrino ranges from 3:1 to $\sim 1: 100$, and for the ${ }^{7} B e$ neutrinos is 1:100 to 1:1000. Using enriched ${ }^{70} \mathrm{Ge}$ or ${ }^{73} \mathrm{Ge}(>85 \%)$ as detector material, the abundance of the $\beta \beta$ emitter can be reduced up to a factor of 1500 . In this case the $p p$-signal is not disturbed by $2 \nu \beta \beta$ decay. The ${ }^{7} B e$ signal to $\beta \beta$ signal ratio will be about 1:1. It can easily be extracted with the help of the seasonal modulation of the flux originating from the earth's excentricity. (The DAMA signal is successfully extracted at a signal to background ratio of $1: 10$ by modulation).

\section{Comparison with other Detector Plans}

GENIUS will allow to look for the $p p$ and ${ }^{7} \mathrm{Be}$ solar neutrinos by elastic neutrino-electron scattering with a threshold (Fig. 2) which would be the lowest threshold among gther proposals to detert $p p$ neutrinos such as HERON 24 , HELLAZ24, NEON24, LENS2624, MOON24, XMASS25, 24.

The counting rate of GENIUS (10 ton) would be 6 events per day for $p p$ and 18 per day for ${ }^{7} \mathrm{Be}$ neutrinos, i.e. similar to BOREXINO, but by a factor of 30 to 60 larger than a 20 ton LENS detector and a factor of 10 larger than 
Table 3. Some key numbers of running and future solar neutrino experiments.

\begin{tabular}{|c|c|c|c|c|c|}
\hline & \multirow[t]{2}{*}{$\begin{array}{c}\text { Threshold } \\
(\mathrm{MeV})\end{array}$} & \multirow[t]{2}{*}{$\begin{array}{l}\text { Resolution } \\
\quad(\mathrm{keV})\end{array}$} & \multirow[t]{2}{*}{$\begin{array}{c}\text { Mass } \\
\text { (tonnes) }\end{array}$} & \multirow[t]{2}{*}{ Reactions } & $\begin{array}{l}\text { Sensitivity } \\
\text { (\%/ev/day) }\end{array}$ \\
\hline & & & & & ${ }^{7} \mathrm{Be},{ }^{8} \mathrm{~B}, \quad p p$ \\
\hline$\frac{\text { Water }}{\text { SUPERK }}$ & 5. & & 50.000 & $\begin{array}{l}\text { Cerenkov } \\
\gamma- \\
\text { rays }\end{array}$ & ${ }^{8} \mathrm{~B}: 100 \% / 20-30 d^{-1}$ \\
\hline$\frac{37 \mathrm{Cl}}{\text { Davis }}$ & .817 & & 615. & $\nu_{e}+{ }^{37} A r \rightarrow$ & $\underbrace{{ }^{7} B e: 20 \% ;{ }^{8} B: 80 \%}$ \\
\hline $\begin{array}{c}\text { Experiment } \\
\text { (absorpt.) }\end{array}$ & & & $\mathrm{C}_{2} \mathrm{Cl}_{4}$ & ${ }^{37} \mathrm{Cl}+e^{-}$ & $\sim 1 d^{-1}$ \\
\hline $\begin{array}{c}{ }^{71} \mathrm{Ga} \\
(\mathrm{GALLE} X) \\
\text { GNO, } \\
\text { SAGE }\end{array}$ & 0.235 & & $\begin{array}{l}30 .+ \\
57 .\end{array}$ & $\begin{array}{c}{ }^{71} G a+\nu_{e} \rightarrow \\
{ }^{71} G e+e^{-}\end{array}$ & $\begin{array}{c}{ }^{7} \mathrm{Be}: 30 \% \\
\underbrace{8} B: 10 \% ; \quad p p: 60 \%\end{array}$ \\
\hline & & & & & $\sim 1 d^{-1}$ \\
\hline$\frac{\mathrm{D}_{2} \mathrm{O}}{\mathrm{SNO}}$ & $\begin{array}{l}1.4(\mathrm{CC}) \\
2.2(\mathrm{NC}) \\
\text { elast. - } 0\end{array}$ & & 1000. & $\begin{array}{c}\nu_{e}+d \rightarrow \\
p+p+e \\
\nu_{x}+d \rightarrow \\
n+p+\nu_{x}\end{array}$ & ${ }^{8} \mathrm{~B}: 100 \%$ \\
\hline $\begin{array}{c}\frac{176 \mathrm{Yb}}{\mathrm{LENS}} \\
\text { (absorpt.) }\end{array}$ & $\begin{array}{l}0.241 \\
0.301\end{array}$ & 100. & $\begin{array}{l}\text { 10.-30. } \\
(8 \%) \text { enr. }\end{array}$ & $\begin{array}{c}{ }^{176} Y b+\nu_{e} \rightarrow \\
{ }^{176} \mathrm{Lu}+e\end{array}$ & $\begin{array}{c}{ }^{7} \text { Be: } 0.2 d^{-1} \\
p p: 0.3 d^{-1}\end{array}$ \\
\hline $\begin{array}{l}\text { G6 Ge } \\
\text { (scatter.) }\end{array}$ & 0.011 & $\begin{array}{l}0.3 \% \text { at } \\
300 \mathrm{keV}\end{array}$ & 1.-10. & $\begin{array}{c}\nu+e^{-} \rightarrow \\
\nu+e^{-}\end{array}$ & $\begin{array}{l}{ }^{7} \mathrm{Be}: 33 \% / .6-6 d^{-1} \\
p p: 66 \% / 1.8-18 d^{-1}\end{array}$ \\
\hline $\begin{array}{c}\text { Scintill. } \\
\text { BOREXINO } * \\
\text { KAMLAND } \dagger \\
\text { HERON } \\
\text { XMASS } \sharp\end{array}$ & $\begin{array}{l}\sim 0.030 \\
0.050 \text { or } \\
0.352\end{array}$ & $4 \% *$ & $\begin{array}{c}100 . * \\
1000 . \dagger \\
10 . \ddagger \\
10 . \sharp\end{array}$ & $\begin{array}{c}\nu+e^{-} \rightarrow \\
\nu+e^{-}\end{array}$ & $\begin{array}{c}{ }^{7} \mathrm{Be}: 60 d^{-1} * \\
{ }^{7} \mathrm{Be}: 6 d^{-1} \sharp \\
p p: 14 d^{-1} \sharp\end{array}$ \\
\hline $\begin{array}{c}\text { Drift } \\
\text { Chambers: } \\
\text { HELLAZ** } \\
\text { (scatter.) } \\
\text { MOON †† } \\
\text { (absorpt.) }\end{array}$ & $\begin{array}{l}0.100 * * \\
0.168 \dagger \dagger\end{array}$ & $\begin{array}{l}5 \% * * \\
7 \% \dagger \dagger\end{array}$ & $\begin{array}{c}2000 \mathrm{~m}^{3} * * \\
3.3 \dagger \dagger\end{array}$ & $\begin{array}{c}\nu+e^{-} \rightarrow \\
\nu+e^{-} * * \\
\nu+{ }^{100} M o \rightarrow \\
{ }^{100} T c+\mathrm{e}\end{array}$ & $\begin{array}{c}{ }^{7} \mathrm{Be}: 4 d^{-1}{ }^{*} * \\
p p: 7 d^{-1}{ }^{* *} \\
{ }^{7} \mathrm{Be}: 0.4 d^{-1} \dagger \dagger \\
\text { pp: } 1.1 d^{-1} \dagger \dagger\end{array}$ \\
\hline
\end{tabular}

the MOON detector (see Table 3).

The good energy resolution for detecting the recoiling electrons would allow for the first time to measure the $1.3 \mathrm{keV}$ predicted shift of the average energy of the beryllium neutrino line. This shift is a direct measure of the 
central temperature of the Sun 37 .

\section{GENIUS and the Neutrino Mass from Double Beta}

Another - more general - contribution to neutrino physics by GENIUS comes from it potential to investigate double beta decay. It has been shown recentlyl, that by double beta experiments it will be possible to test almost all neutrino mass scenarios allowed by the present neutrino oscillation experiments.

This is shown in Fig. 7 for the Large Mixing Angle and LOW MSW solutions, presently favored by solar neutrino oscillation experiments. Nature seems to be generous to us, that with an increase of the sensitivity for the effective neutrino mass $\langle m\rangle$ - the observable of double beta decay - by two orders of magnitude compared to the present best limit (HEIDELBERG-MOSCOW experiment14 ) almost the full range of expectations from oscillation experiments can be covered. This is possible, however, only by exploiting this method to obtain information on the neutrino mass to the ultimate limit.

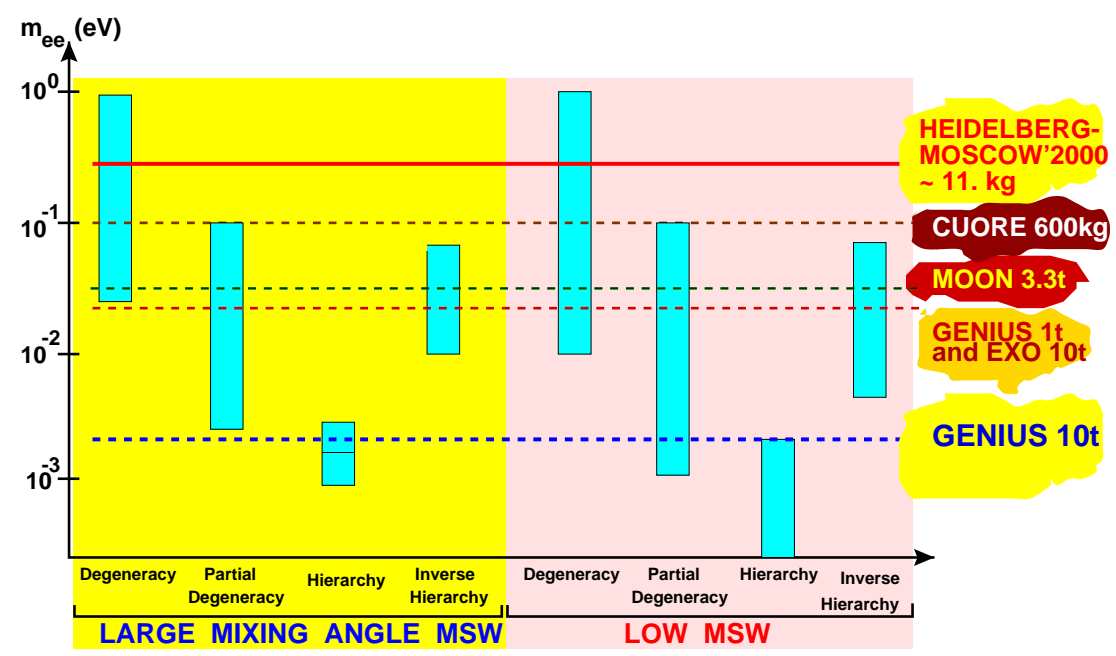

Figure 7. Summary of values for $m_{e e}=\langle m\rangle$ expected from neutrino oscillation experiments (status NEUTRINO2000), in the two neutring mass schemes presently favored by solar $\nu$ experiments. For a more general analysis seet. The expectations are compared with the recent neutrino mass limits obtained from the HEIDELBERG-MOSCOW experiment 14,21 as well as the expected sensitivitieffor the CUORE, AON, EXO proposals and the 1 ton and 10 ton proposal of GENIUS17. 18 (for details see 13 ). 
This can be done by the GENIUS project (for details see明㫜).

\section{GENIUS and Cold Dark Matter Search}

GENIUS is also the only of the new projects under discussion (see Tabl. 4) which simultaneously with its potential for real-time detection of low-energy neutrinos, and for double beta decay, has a huge potential for cold dark matter search.

Table 4. Some of the new projects under discussion for future double beta decay experiments (see ref.3).

\begin{tabular}{|c|c|c|c|c|}
\hline & NEW & \multicolumn{2}{|c|}{ PROJECTS } & \multirow[b]{2}{*}{$\begin{array}{c}\text { POTENTIAL } \\
\text { FOR S,OLAR } \\
\nu^{\prime} \mathrm{S} \\
\end{array}$} \\
\hline & $\begin{array}{l}\text { BACKGROUND } \\
\text { REDUCTION }\end{array}$ & $\begin{array}{c}\text { MASS } \\
\text { INCREASE }\end{array}$ & $\begin{array}{c}\text { POTENTIAL } \\
\text { FOR DARK } \\
\text { MATTER } \\
\end{array}$ & \\
\hline GENIUS & + & + & + & $\left.t^{*}\right)$ \\
\hline XMASS & + & + & + & $\left.+^{*}\right)$ \\
\hline CUORE & $(+)$ & + & - & - \\
\hline MOON & $(+)$ & + & - & + \\
\hline EXO & + & + & - & - \\
\hline MAJORANA & - & + & - & - \\
\hline$*)$ & $\begin{array}{l}\text { real time mea } \\
\text { with thresh }\end{array}$ & surement & $\begin{array}{l}\text { of pp neut } \\
50 \mathrm{keV}(! !)\end{array}$ & inos \\
\hline
\end{tabular}

Already now the HEIDELBERG-MOSCOW experiment is the prost sensitive Dark Matter experiment worldwide concerning the raw data 10 [1], 3 . GENIUS would already in a first step, with $100 \mathrm{~kg}$ of natural Ge detectors, cover a significant part of the MSSM parameter space for prediction of neutralinos as cold dark matter (Fig. 8) (see, e.g. 30). For this purpose the background in the energy range $<100 \mathrm{keV}$ has to be reduced to $10^{-2}$ events/(kg y keV), which is possible if the detectors are produced and handled on Earth surface under heavy shielding, to reduce the cosmogenic background produced by spallation through cosmic radiation (critical products are tritium, ${ }^{68} \mathrm{Ge},{ }^{63} \mathrm{Ni}, \ldots$ ) to a minimum. For details we refer to 18 .22.16. Fig. A shows that at this level solar neutrinos as source of background are still negligible. Fig. 8 shows together with the expected sensitivity of GENIUS, for this background, predictions 


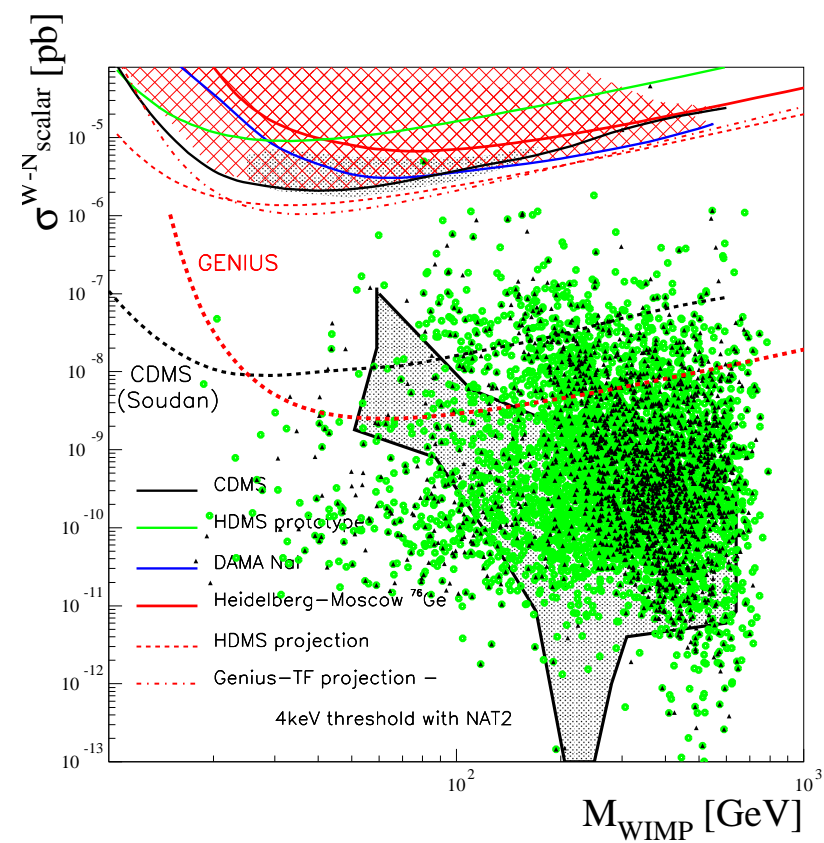

Figure 8. WIMP-nucleon cross section limits in pb for scalar interactions as function of the WIMP mass in GeV. Shown are contour lines of present experimental limits (solid lines) and of projected experiments (dashed lines). Also shown is the region of evidence published by DAMA. The troretical expectations are shown by a scatter plot (from 31 ) and by the grey region (from 33 ). Only GENIUS will be able to probe the shown range also by the signature from seasonal modulations.

for neutralinos as dark matter by two models, one basing on supergravity 33 , another starting from more relaxed unification conditions31,32.

The sensitivity of GENIUS for Dark Matter corresponds to that obtainable with a $1 \mathrm{~km}^{3}$ AMANDA detector for indirect detection (neutrinos from annihilation of neutralinos captured at the Sun) (see34). Interestingly both experiments would probe different neutralino compositions: GENIUS mainly gaugino-dominated neutralinos, AMANDA mainly neytralinos with comparable gaugino and Higgsino components (see Fig. 38 in 34 ). It should be stressed that, together with DAMA, GENIUS will be the only future Dark Matter experiment, which would be able to positively identify a dark matter signal by the seasonal modulation signature. This cannot be achieved, for example, by the CDMS experiment. 


\section{Conclusion}

Concluding, GENIUS could measure solar pp neutrinos in real time. The detector reaction would be elastic neutrino-electron scattering, with a threshold of 11 or at most $19 \mathrm{keV}$ (limit of possible tritium background), which would be the lowest threshold among other proposals, to detect $p p$ neutrinos, such as HERON24, HELLAZ24, NEON24, LENS2624, MOON24, XMASSE5 24.

The counting rate of GENIUS (10 ton) of 6 events per day for $p p$ and 18 per day for ${ }^{7} \mathrm{Be}$ neutrinos, would be similar to BOREXINO, but by a factor of 30-60 larger than a 20 ton LENS detector and a factor of 10 larger than the MOON datector (see Table 3).

The good energy resolution for detecting the recoiling electrons would allow for the first time to measure the $1.3 \mathrm{keV}$ predicted shift of the average energy of the beryllium neutring line. This shift is a direct measure of the central temperature of the Sun 37 .

GENIUS would further allow - in its main applications - to probe, by search for double beta decay, almost the full range of expectations for the effective neutrino mass in practically all neutrino mass scenarios, consistent with present neutrino oscillation experiments. Finally it would be the most sensitive cold dark matter detector, having the potential to probe a significant part of the MSSM parameter space for prediction of neutralinos as cold dark matter. It will be the only future dark matter experiment (besides DAMA),

which would be able to positively by identify a dark matter signal by the seasonal modulation signature.

\section{References}

1. H.V. Klapdor-Kleingrothaus, H. Päs and A.Yu. Smirnov, Preprint: hepph/0003219, (2000) and in Phys. Rev. D (2000).

2. H.V. Klapdor-Kleingrothaus, H. Päs and A.Yu. Smirnov, in Proc. of DARK2000, Heidelberg, 10-15 July, 2000, Germany, ed. H.V. Klapdor-Kleingrothaus, Springer, Heidelberg (2001) and Preprint: hepph/0103076 .

3. H.V. Klapdor-Kleingrothaus, "60 Years of Double Beta Decay", World Scientific, Singapore (2001) 1253 p.

4. H.V. Klapdor-Kleingrothaus, H. Päs, Preprint: physics/0006024 and Comm. in Nucl. and Part. Phys. (2000).

5. J. Hellmig and H.V. Klapdor-Kleingrothaus, Z. Phys. A 359, 351 (1997).

6. L. Baudis, G. Heusser, B. Majorovits, Y. Ramachers, H. Strecker and H.V. Klapdor-Kleingrothaus, hep-ex/ 9811040 and Nucl. Instr. Meth. 
A 426, 425 (1999).

7. L. Baudis and H.V. Klapdor-Kleingrothaus, Eur. Phys. J. A 5, 441-443 (1999) and in Proceedings of the 2nd Int. Conf. on Particle Physics Beyond the Standard Model BEYOND'99, Castle Ringberg, Germany, 6-12 June 1999, edited by H.V. Klapdor-Kleingrothaus and I.V. Krivosheina, IOP Bristol, 1023 - 1036 (2000).

8. H.V. Klapdor-Kleingrothaus et al., to be publ. 2001 and http ://www.mpi-hd.mpg.de/non_acc /

9. HEIDELBERG-MOSCOW Collaboration, Phys. Rev. D 59, 022001 (1998).

10. L. Baudis, A. Dietz, B. Majorovits, F. Schwamm, H. Strecker and H.V. Klapdor-Kleingrothaus, Phys. Rev. D 63，022001 (2000) and astroph/0008339.

11. Y. Ramachers for the CRESST Collaboration in Proc. of XIth Rencontres de Blois, Frontiers of Matter, France, June 27-July 3, 1999.

12. H.V. Klapdor-Kleingrothaus et al. Proc. DARK2000, Heidelberg, Germany, July 10-15, 2000, Ed. H.V. Klapdor-Kleingrothaus, Springer, Heidelberg (2001).

13. H.V. Klapdor-Kleingrothaus et al. in Proc. of Third International Conference on Dark Matter in Astro and Particle Physics, DARK2000, Heidelberg, Germany, July 10-15, 2000, Springer, Heidelberg (2001), ed. H.V. Klapdor-Kleingrothaus and Preprint: hep-ph/0103082.

14. H.V. Klapdor-Kleingrothaus et al. in Proc. of Third International Conference on Dark Matter in Astro and Particle Physics, DARK2000, Heidelberg, Germany, July 10-15, 2000, Springer, Heidelberg (2001), ed. H.V. Klapdor-Kleingrothaus and Preprint: hep-ph/0103062, subm. to Phys. Rev. D (2001).

15. H.V. Klapdor-Kleingrothaus, In Proc. of International Workshop Non-Accelerator New Physics in neutrino observations, NANPino-2000, Dubna, July 19-22, 2000, ed. V. Bednyakov et al., to be publ. in Particles and Nuclei, Letters, issues 1/2 (2001) and Preprint: hep-ph/0102319.

16. H.V. Klapdor-Kleingrothaus and B. Majorovits, in Proc. of 3rd International Workshop on the Identification of Dark Matter (IDM2000), York, England, 18-22 Sep 2000, World Scientific, Singapore (2001) and Preprint: hep-ph/0103079.

17. H.V. Klapdor-Kleingrothaus in Proceedings of BEYOND'97, First International Conference on Particle Physics Beyond the Standard Model, Castle Ringberg, Germany, 8-14 June 1997, edited by H.V. KlapdorKleingrothaus and H. Päs, IOP Bristol 485-531 (1998)

18. H.V. Klapdor-Kleingrothaus et al. MPI-Report MPI-H-V26-1999 and 
Preprint: hep-ph/9910205 and in Proceedings of the 2nd Int. Conf. on Particle Physics Beyond the Standard Model BEYOND'99, Castle Ringberg, Germany, 6-12 June 1999, edited by H.V. Klapdor-Kleingrothaus and I.V. Krivosheina, IOP Bristol, 915 - 1014 (2000).

19. H.V. Klapdor-Kleingrothaus, in Proc. of 18th Int. Conf. on Neutrino Physics and Astrophysics (NEUTRINO 98), Takayama, Japan, 4-9 Jun 1998, (eds) Y. Suzuki et al. Nucl. Phys. Proc. Suppl. 77, $357-368$ (1999).

20. H.V. Klapdor-Kleingrothaus, in Proc. of WEIN'98, "Physics Beyond the Standard Model", Proceedings of the Fifth Intern. WEIN Conference, P. Herczeg, C.M. Hoffman and H.V. Klapdor-Kleingrothaus (Editors), World Scientific, Singapore, 275-311 (1999).

21. H.V. Klapdor-Kleingrothaus et al., Annual Report Gran Sasso 2000 (2001).

22. H.V. Klapdor-Kleingrothaus, in Proc. of Int. Conference NOW2000 "Origins of Neutrino Oscillations", Nucl. Phys. B (2001) ed. G. Fogli and Preprint: hep-ph/0102277, Preprint: hep-ph/0102276.

23. H.V. Klapdor-Kleingrothaus, in Proc. of NOON2000, International Workshop on "Neutrino Oscillations and Their Origin", Tokyo, 6-18 Dec. 2000, World Scientific, Singapore (2001) and Preprint: hep-ph/0103074.

24. Proc. Int. Workshop on Low Energy Solar Neutrinos, LowNu2, December 4 and 5 (2000) Tokyo, Japan, ed: Y. Suzuki, World Scientific, Singapore (2001), home page: http://www-sk.icrr.utokyo.ac.jp/neutlowe/2/transparency index.html

25. Y. Suzuki for the collaboration, Preprint: hep-ph/0008296.

26. M. Fujiwara et al., Phys. Rev. Lett. 85, 4442-4445 (2000) and Preprint: nucl-ex/0006006: M. Bhattacharya et al. Phys. Rev. Lett. 85, 44464449 (2000) and Preprint: nucl-ex/0006005.

27. H.V. Klapdor-Kleingrothaus, J. Hellmig and M. Hirsch, J. Phys. G 24, 483 (1998).

28. H.V. Klapdor-Kleingrothaus, Int. J. Mod. Phys. A 13, 3953 (1998).

29. H.V. Klapdor-Kleingrothaus, Springer Tracts in Modern Physics, 163, 69-104 (2000), Springer-Verlag, Heidelberg, Germany (2000).

30. H.V. Klapdor-Kleingrothaus and Y. Ramachers, Eur. Phys. J. A 3, 85-92 (1998).

31. V.A. Bednyakov and H.V. Klapdor-Kleingrothaus, Phys. Rev. D 62 (2000) 043524/1-9 and hep-ph/9908427.

32. V.A. Bednyakov and H.V. Klapdor-Kleingrothaus, Preprint: hepph/0011233 (2000) in press in Phys. Rev. D (2001).

33. J. Ellis, A. Ferstl and K.A. Olive, Phys. Lett. B 481, 304-314 (2000) 
and Preprint: hep-ph/0001005 and Preprint: hep-ph/0007113.

34. J. Edsjö, Neutralinos as dark matter - can we see them? Seminar given in the theory group, Department of Physics, Stockholm University, October 12, 1999, home page: http://www.physto.se/edsjo

35. see: http://www.sns.ias.edu.jnb

36. J.N. Bahcall, Neutrino Astrophysics, Cambridge Univ. Press (1989).

37. J.N. Bahcall, Phys. Rev Lett. 71, 2369 (1993).

38. J.N. Bahcall and P.I. Krastev, Phys. Rev. C 56, 2839 (1997).

39. J.N. Bahcall, S. Basu and M. Pinsonneault, Phys. Lett. B 433, 1 (1998). 